\title{
Matrix metalloproteinase activation by free neutrophil elastase contributes to bronchiectasis progression in early cystic fibrosis
}

\author{
Luke W. Garratt ${ }^{1,2}$, Erika N. Sutanto ${ }^{2,3}$, Kak-Ming Ling ${ }^{2}$, Kevin Looi ${ }^{1}$, \\ Thomas losifidis ${ }^{1,4}$, Kelly M. Martinovich², Nicole C. Shaw ${ }^{2}$, \\ Elizabeth Kicic-Starcevich ${ }^{2,3}$, Darryl A. Knight ${ }^{5,6,7}$, Sarath Ranganathan ${ }^{8,9,10}$, \\ Stephen M. Stick ${ }^{1,2,3,4}$ and Anthony Kicic ${ }^{1,2,3,4}$ on behalf of the Australian \\ Respiratory Early Surveillance Team for Cystic Fibrosis (AREST CF) 2,3,9,10,11
}

\begin{abstract}
Affiliations: 'School of Paediatrics and Child Health, University of Western Australia, Perth, Australia. ${ }^{2}$ Telethon Kids Institute, University of Western Australia, Perth, Australia. ${ }^{3}$ Dept of Respiratory Medicine, Princess Margaret Hospital for Children, Perth, Australia. ${ }^{4}$ Centre for Cell Therapy and Regenerative Medicine, School of Medicine and Pharmacology, University of Western Australia, Perth, Australia. ${ }^{5}$ School of Biomedical Sciences and Pharmacy, University of Newcastle, Callaghan, Australia. ${ }^{6}$ Priority Research Centre for Asthma and Respiratory Disease, Hunter Medical Research Institute, Newcastle, Australia. ${ }^{7}$ Dept of Anesthesiology, Pharmacology and Therapeutics, University of British Columbia, Vancouver, BC, Canada. ${ }^{8}$ Dept of Respiratory Medicine, Royal Children's Hospital, Melbourne, Australia. ${ }^{9}$ Murdoch Childrens Research Institute, Melbourne, Australia. ${ }^{10}$ Dept of Paediatrics, University of Melbourne, Melbourne, Australia. ${ }^{11} \mathrm{~A}$ full list of the AREST CF members and their affiliations can be found in the Acknowledgements section.
\end{abstract}

Correspondence: Anthony Kicic, Telethon Kids Institute, Subiaco, Perth, 6008, Western Australia, Australia. E-mail: Anthony.Kicicatelethonkids.org.au

ABSTRACT Neutrophil elastase is the most significant predictor of bronchiectasis in early-life cystic fibrosis; however, the causal link between neutrophil elastase and airway damage is not well understood. Matrix metalloproteinases (MMPs) play a crucial role in extracellular matrix modelling and are activated by neutrophil elastase. The aim of this study was to assess if MMP activation positively correlates with neutrophil elastase activity, disease severity and bronchiectasis in young children with cystic fibrosis.

Total MMP-1, MMP-2, MMP-7, MMP-9, tissue inhibitor of metalloproteinase (TIMP)-2 and TIMP-1 levels were measured in bronchoalveolar lavage fluid collected from young children with cystic fibrosis during annual clinical assessment. Active/pro-enzyme ratio of MMP-9 was determined by gelatin zymography. Annual chest computed tomography imaging was scored for bronchiectasis.

A higher MMP-9/TIMP-1 ratio was associated with free neutrophil elastase activity. In contrast, MMP2/TIMP-2 ratio decreased and MMP-1 and MMP-7 were not detected in the majority of samples. Ratio of active/pro-enzyme MMP-9 was also higher in the presence of free neutrophil elastase activity, but not infection. Across the study cohort, both MMP-9/TIMP-1 and active MMP-9 were associated with progression of bronchiectasis.

Both MMP-9/TIMP-1 and active MMP-9 increased with free neutrophil elastase and were associated with bronchiectasis, further demonstrating that free neutrophil elastase activity should be considered an important precursor to cystic fibrosis structural disease.

@ERSpublications

In young children with CF, activation of MMP-9 by free NE may provide one mechanism driving structural lung disease http://ow.ly/KArB5

This article has supplementary material available from erj.ersjournals.com

Received: Nov 162014 | Accepted after revision: March 042015 | First published online: April 302015

Copyright OERS 2015 


\section{Introduction}

Prevention of air trapping and bronchiectasis is key to improving quality and longevity of life for children with cystic fibrosis [1]. There is now a strong body of evidence that these key structural abnormalities begin in infancy $[2,3]$. A number of studies that have examined bronchoalveolar lavage fluid (BALF) report dysregulated inflammation that begins as early as 3 months and is characterised by extensive neutrophil infiltration into the airway $[2,4]$. Within this inflammatory milieu, free neutrophil elastase activity is a strong predictor of bronchiectasis [5]. Direct mechanisms linking neutrophil elastase to bronchiectasis have not been identified, although the currently accepted proposition is that chronic neutrophil elastase degrades airway extracellular matrix (ECM) and prevents adequate tissue repair, resulting in abnormal airway remodelling [6].

In addition to neutrophil elastase, other proteases known to modify lung tissue such as matrix metalloproteinases (MMPs) could contribute to the progression of cystic fibrosis lung disease [7]. These enzymes can degrade all ECM components, therefore they are carefully regulated in repairing tissues and are not usually detectable in healthy lung tissue [8]. The activity of secreted MMPs is regulated by both an inhibitory protein residue that requires removal for functional activity, as well as inhibition by 1:1 binding of appropriate tissue inhibitors of matrix metalloproteinases (TIMPs) [9]. Of the MMP family, the gelatinases MMP-2 and MMP-9 have previously been reported to correlate with exacerbations, sputum viscosity and worse respiratory outcomes in adolescents and adults with cystic fibrosis [10-15]. Studies indicate that neutrophil elastase cleaves the inhibitory protein residue on several MMPs, including MMP-2 and MMP-9 $[13,16-18]$, and might also degrade TIMP-1 [13, 18]. These data suggest that chronic free neutrophil elastase in the cystic fibrosis airway can augment MMP-9 activation and reduce MMP inhibition, leading to increased, persistent MMP activity in the airway, and thus contribute to airway remodelling.

We propose that as a consequence of airway inflammation in early life, disruption of MMP regulation during the course of repair is a possible mechanism that links neutrophil elastase with abnormal remodelling. However, there are only limited data regarding MMP expression patterns and their activation profiles in the first years of life $[14,19]$, when both neutrophilic inflammation and airway remodelling first become apparent. The present study tested the hypothesis that expression and activation patterns of MMP-2 and MMP-9 positively correlate with disease severity in infants and young children with cystic fibrosis.

\section{Materials and methods}

The online supplementary material includes full details of materials and methods.

\section{Subjects}

This study was approved by the human ethics committees at the Princess Margaret Hospital for Children (Perth, Australia) and the Royal Children's Hospital (Melbourne, Australia). Cystic fibrosis transmembrane conductance regulator genotype was determined as part of newborn screening. Included were 64 BALF samples from 61 children with cystic fibrosis, collected during their clinically directed annual surveillance visit $[2,3]$. Samples of BALF were obtained from the right middle lobe, under general anaesthesia, during bronchoscopy according to the recommendations of the European Respiratory Society Task Force on bronchoalveolar lavage in children [20]. Bronchiectasis was detected by computed tomography (CT) and scored as previously described $[3,5]$.

\section{BALF processing}

The first BALF aspirate was analysed for viral, bacterial and fungal pathogens [2, 3]. Inflammatory markers, neutrophil count, interleukin- 8 and neutrophil elastase were measured in the pooled second and third BALF aspirates, as previously described [21]. Expression of MMP-1, MMP-2, MMP-7, MMP-9, TIMP-1 and TIMP-2 were measured using sandwich ELISA (RayBiotech, Norcross, GA, USA) (detection limits $24 \mathrm{pg} \cdot \mathrm{mL}^{-1}, 7 \mathrm{ng} \cdot \mathrm{mL}^{-1}, 138 \mathrm{pg} \cdot \mathrm{mL}^{-1}, 10 \mathrm{pg} \cdot \mathrm{mL}^{-1}, 80 \mathrm{pg} \cdot \mathrm{mL}^{-1}$ and $20 \mathrm{pg} \cdot \mathrm{mL}^{-1}$, respectively) in the pooled aspirate. Samples below detection range were arbitrarily reported as half the lower limit to minimise the difficulties associated with statistical analysis of zero values, as previously described [22].

Support statement: Funding for the AREST CF programme was obtained from the Cystic Fibrosis Foundation Therapeutics (SLY04A0, STICK09A0), the National Health and Medical Research Council of Australia (NHMRC) (centre of research excellence \#1000896) and project grant funding from the NHMRC and Cystic Fibrosis Australia (NHMRC 1043768). S.M. Stick is a NHMRC Practitioner Fellow. L.W. Garratt is funded by a PhD scholarship from the NHMRC and supplementary scholarships from the University of Western Australia and Cystic Fibrosis Western Australia. This research was presented in part at the Australasian Cystic Fibrosis Conference and the North American Cystic Fibrosis Conference with support from the University of Western Australia. Funding information for this article has been deposited with FundRef.

Conflict of interest: Disclosures can be found alongside the online version of this article at erj.ersjournals.com 
Activation of pro-MMP-9 by neutrophil elastase

Activation of pro-MMP-9 by neutrophil elastase was assessed by incubating $200 \mathrm{ng} \cdot \mathrm{mL}^{-1}$ recombinant human MMP-9 (RayBiotech) with an equal volume of $200 \mathrm{nM}$ purified human sputum neutrophil elastase (Elastin Products Company, Owensville, MO, USA) for $30 \mathrm{~min}$ at $37^{\circ} \mathrm{C}$.

\section{Gelatin zymography}

Activation of MMP-2 and MMP-9 in BALF was assessed by gelatin zymography, as previously described [23], with the following modifications. A $2 \times$ nonreducing loading buffer was used; gels were electrophoresed at $200 \mathrm{mV}$ for $120 \mathrm{~min}$ at $4^{\circ} \mathrm{C}$; the developing buffer consisted of $200 \mathrm{nM} \mathrm{NaCl}, 10 \mathrm{mM}$ Trizma base (Sigma-Aldrich, St Louis, MO, USA), $40 \mathrm{mM}$ Trizma hydrochloride (Sigma-Aldrich), $6.67 \mathrm{mM}$ $\mathrm{CaCl}_{2}$ and $0.02 \%(\mathrm{v} / \mathrm{v})$ Brij-35 (Sigma-Aldrich); and the staining buffer consisted of $0.5 \%(\mathrm{v} / \mathrm{v})$ Coomassie blue (Sigma-Aldrich) in 50\% (v/v) methanol, 20\% (v/v) acetic acid and 30\% (v/v) $\mathrm{dH}_{2} \mathrm{O}$. To confirm that the bands observed were MMP-9, gels were incubated under identical conditions with the addition of $10 \mu \mathrm{M}$ MMP-9-specific inhibitor I (ab14180; Abcam, Cambridge, UK) or $10 \mu \mathrm{M}$ broad-spectrum MMP inhibitor GM6001 (Abcam).

\section{Statistical analysis}

Data were tested for population normality as well as homogeneity of variance by histogram plots and D’Agostino-Pearson omnibus test. Normally distributed data were reported as mean \pm sD and non-normally distributed data were reported as median (interquartile range). Tests with appropriate parametric or nonparametric statistical analyses were conducted using GraphPad Prism 5 (GraphPad Software, La Jolla, CA, USA), with p-values $<0.05$ considered to be significant. Exact p-values are reported, except for multiple comparisons, where multiplicity-adjusted p-values are reported.

\section{Results}

To assess the relationship of neutrophil elastase and MMP in early life, 32 BALF samples were randomly selected from the pool of Australian Respiratory Early Surveillance Team for Cystic Fibrosis (AREST CF) samples previously determined to have detectable free neutrophil elastase. Another 32 samples with no neutrophil elastase were then age matched. Study demographics are detailed in table 1. Patient ages ranged from 0.16 to 7.09 years; 33 samples were from Phe508del homozygous patients; and bronchiectasis was identified in 41 subjects. Respiratory infection was classified as $\geqslant 10^{4}$ bacterial CFU.mL ${ }^{-1}$ BALF; 31 samples were defined as infected, with 13 samples positive for presence of Aspergillus species and eight samples positive for presence of Pseudomonas aeruginosa at the time of sampling (table 2).

The expression and activation of individual MMPs and TIMPs were dependent upon the presence of pathogens and neutrophil elastase in BALF (table 3). Median concentrations of MMP-2 were decreased in the presence of neutrophil elastase (8.15 versus $\left.3.5 \mathrm{ng} \cdot \mathrm{mL}^{-1}, \mathrm{p}<0.0001\right)$ (table 3 ), and only two BALF samples with free neutrophil elastase had measurable MMP-2 activity. In contrast, the majority of BALF samples (52 out of $64 ; 81.25 \%$ ) had detectable MMP-9, with a mean expression 280 -fold that of MMP-2. Significant elevation of total MMP-9 was observed in the presence of infection (median 465.7 versus $1406 \mathrm{ng} \cdot \mathrm{mL}^{-1}, \mathrm{p}=0.0018$ ) (table 3) and free neutrophil elastase (median 269.5 versus $1494 \mathrm{ng} \cdot \mathrm{mL}^{-1}$, $\mathrm{p}<0.0001$ ) (table 3 ). Concentrations of MMP inhibitors did not vary during infection, but both TIMPs were higher in airways with free neutrophil elastase (median TIMP-1 14.40 versus $19.91 \mathrm{ng} \cdot \mathrm{mL}^{-1}, \mathrm{p}=0.0406$, and

TABLE 1 Study demographics

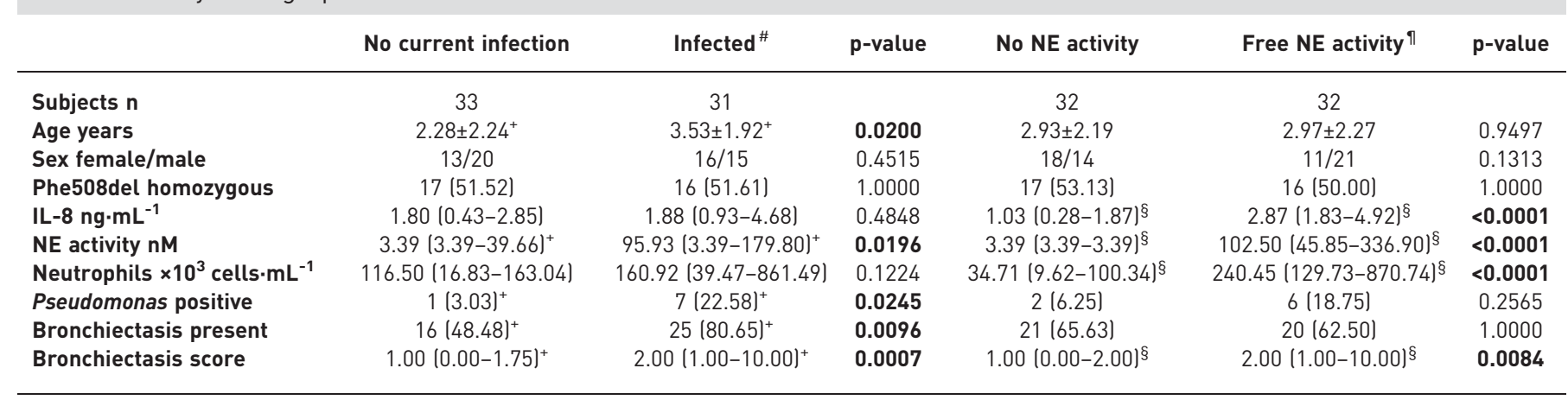

Data are presented as $\mathrm{n}$, mean $\pm \mathrm{SD}, \mathrm{n}(\%)$ or median (interquartile range). Values in bold are statistically significant. NE: neutrophil elastase; IL:

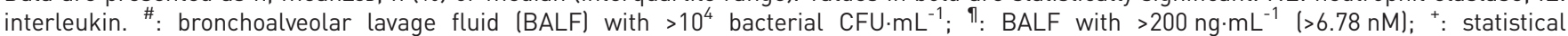
significance between no current infection and infected; ${ }^{\S}$ : statistical significance between no NE activity and free NE activity. 
TABLE 2 Microbiological profiles of the study population

\section{Subject}

None

Mixed oral flora

Aspergillus fumigatus

None

None

None

None

None

Mixed oral flora

Mixed oral flora

None

Mixed oral flora

Pseudomonas aeruginosa

None

Mixed oral flora

None

None

None

None

None

None

Aspergillus fumigatus

None

None

Mixed oral flora

None

None

None

None

Mixed oral flora

Mixed oral flora

None

None
Infected ${ }^{n} n=31$

Mixed oral flora

Aspergillus fumigatus

Aspergillus fumigatus

Mixed oral flora

Pseudomonas aeruginosa

Mixed oral flora

Mixed oral flora

Aspergillus fumigatus

Haemophilus influenzae, Haemophilus species, Staphylococcus aureus

Aspergillus fumigatus, Pseudomonas aeruginosa

Aspergillus fumigatus, Pseudomonas aeruginosa, Scedosporium apiospermum Aspergillus fumigatus

Haemophilus influenzae, Haemophilus species

Mixed oral flora

Staphylococcus aureus

Haemophilus influenzae, Streptococcus pneumoniae

Haemophilus influenzae, Pseudomonas aeruginosa Mixed oral flora

Adenovirus, Aspergillus niger, Haemophilus influenzae

Mixed oral flora

Mixed oral flora

Escherichia coli

Haemophilus influenzae, mixed oral flora

Aspergillus fumigatus, Candida species, Pseudomonas aeruginosa Haemophilus influenzae

Aspergillus flavus, Pseudomonas aeruginosa

Mixed oral flora, Pseudomonas aeruginosa

Aspergillus fumigatus, Stenotrophomonas maltophilia

Achromobacter xylosoxidans, Aspergillus fumigatus

Candida species, Staphylococcus aureus

Candida species

Subjects are ordered by increasing age. Bold text indicates that the subject had free neutrophil elastase activity. None indicates no pathogens could be cultured. " : all pathogens were reported as $10^{3} \mathrm{CFU} \cdot \mathrm{mL}^{-1} ;{ }^{n}: \geqslant 10^{4} \mathrm{CFU} \cdot \mathrm{mL}^{-1}$.

TABLE 3 Expression patterns of matrix metalloproteinases (MMPs) during infection and free neutrophil elastase (NE) activity in very young children with cystic fibrosis

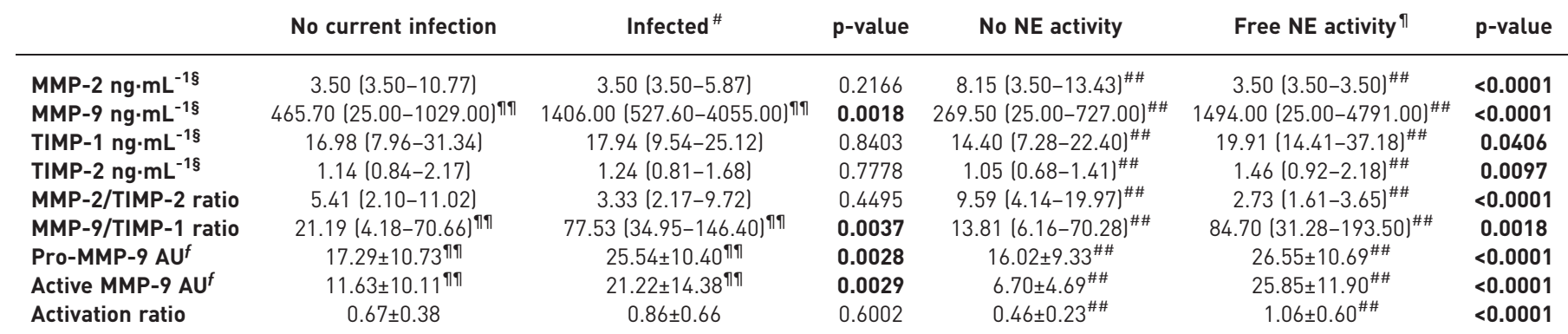

Data are presented as median (interquartile range) or mean \pm SD. Bold text represents statistical significance. TIMP: tissue inhibitor of metalloproteinase; AU: arbitrary units. \#: bronchoalveolar lavage fluid (BALF) with $>10^{4}$ bacterial CFU. $\mathrm{mL}^{-1}:{ }^{\uparrow}: \mathrm{BALF}>200 \mathrm{ng} \cdot \mathrm{mL}^{-1}(>6.78 \mathrm{nM})$ : $\S_{\text {: }}$ samples below detection range were reported as half the lower limit; ${ }^{f}$ : integrated intensity of MMP band as AU; ${ }^{\# \#}$ : statistical significance between no NE activity and free NE activity; ${ }^{\text {ๆा: }}$ statistical significance between no current infection and infected. 
median TIMP-2 1.05 versus $1.46 \mathrm{ng} \cdot \mathrm{mL}^{-1}, \mathrm{p}=0.0097$ ) (table 3). The ratio of total MMP to total respective TIMP provides an approximate measure of MMP regulation in vivo, whereby higher ratios represent reduced inhibition of MMPs. In the presence of free neutrophil elastase, median MMP-9/TIMP-1 ratio was significantly increased (13.81 versus $84.70, \mathrm{p}=0.0018$ ) (table 3), whereas median MMP-2/TIMP-2 ratio was decreased $(9.59$ versus $2.73, \mathrm{p}<0.0001$ ) (table 3 ). In a subset of 32 BALF samples, the collagenase MMP-1 was not detected and MMP-7 levels were minimal, with only six samples featuring detectable MMP-7 (online supplementary table S1).

Activation of MMPs was then assessed by gelatin zymography. First, incubation of recombinant human pro-MMP-9 with $100 \mathrm{nM}$ neutrophil elastase resulted in the appearance of a $\sim 78-\mathrm{kDa}$ active form (fig. 1a), confirming previous findings $[13,18]$. The appearance of an additional $\sim 170-\mathrm{kDa}$ band may be due to activation of a $\sim 220-\mathrm{kDa}$ dimer MMP-9 by neutrophil elastase. Three BALF samples exhibited similar bands with slightly lower migration mobility, as described elsewhere [24]. Gelatinolytic bands $\sim 65 \mathrm{kDa}$ in size (fig. 1a) were identified as MMP-9 lacking the carboxy-terminal domain [25], rather than MMP-2, since no bands developed in the presence of $10 \mu \mathrm{M}$ MMP-9 inhibitor ab142180 or $10 \mu \mathrm{M}$ MMP inhibitor GM6001, and ELISA measurements of total MMP-2 suggest that the amount of sample loaded would be very close to the 10-pg lower limit of zymography sensitivity [26]. As a result, only activation of MMP-9 was assessed in this study, by measuring the intensity of the $\sim 92-\mathrm{kDa}$ pro-MMP-9 and $\sim 78-\mathrm{kDa}$ active MMP-9 bands as arbitrary units (AU) (fig. 2b). Elevated levels of pro-MMP-9 (16.02 \pm 9.33 versus $26.55 \pm 10.69 \mathrm{AU}, \mathrm{p}<0.0001)$ (table 3$)$ and active MMP-9 $(6.70 \pm 4.69$ versus $25.85 \pm 11.90 \mathrm{AU}, \mathrm{p}<0.0001)$ (table 3) were seen in BALF with free neutrophil elastase. Ratio of active MMP-9/pro-MMP-9 did not significantly change during infection $(0.67 \pm 0.38$ versus $0.86 \pm 0.66, \mathrm{p}=0.60)$, however, was increased in the presence of neutrophil elastase $(0.46 \pm 0.23$ versus $1.06 \pm 0.60, \mathrm{p}<0.0001)$ (table 3$)$ and a ratio $>1$ was only seen in BALF with free neutrophil elastase (fig. 1b).
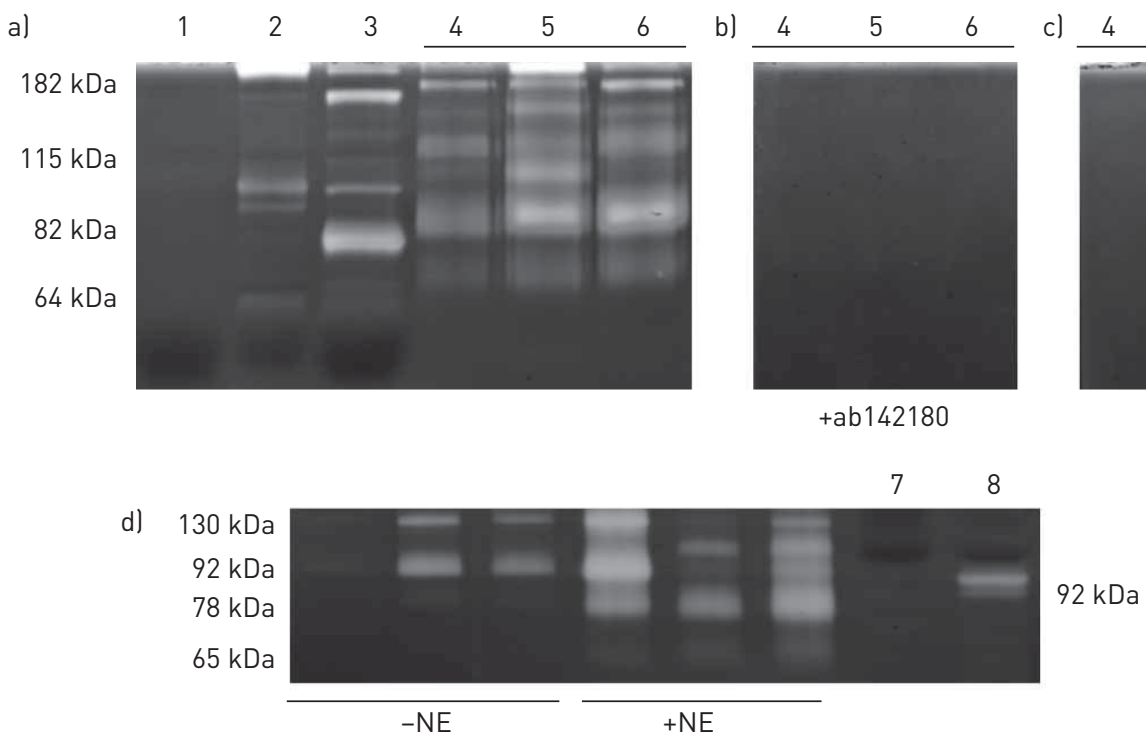

7

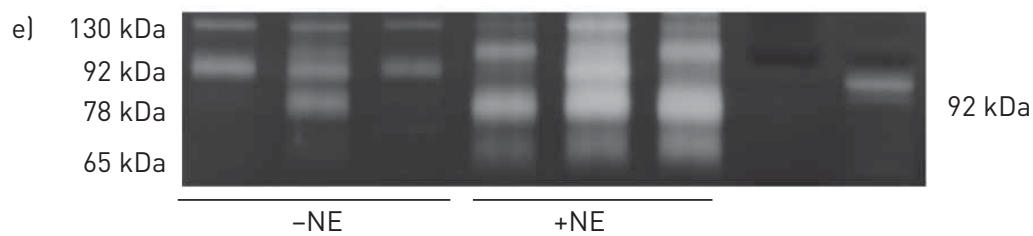

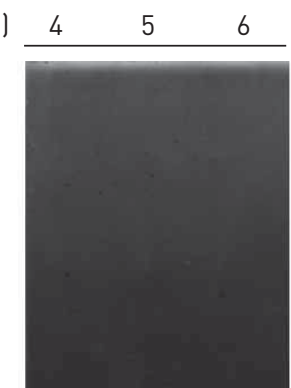

$+a b 142180$
$2 \mathrm{kDa}$

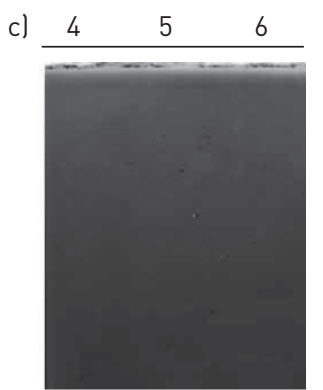

+GM6001

FIGURE 1 Identification and comparison of matrix metalloproteinase (MMP)-9 bands during early-life cystic fibrosis disease. Molecular mass markers are indicated. a) Incubation of purified human neutrophil elastase (NE; lane 1) with recombinant human MMP-9 (lane 2) for $30 \mathrm{~min}$ at $37^{\circ} \mathrm{C}$ results in a $\sim 78-\mathrm{kDa}$ isoform of MMP-9 (lane 3). Similar bands were observed in bronchoalveolar lavage fluid (BALF) from children with cystic fibrosis (lanes 4-6). No bands were observed when gels were developed in the presence of b) MMP-9 inhibitor ab142180 or c) broad-spectrum MMP inhibitor GM6001. d) and e) Compared to cystic fibrosis BALF with no free neutrophil elastase (-NE), density of the $\sim 78-\mathrm{kDa}$ isoform was found to be increased in samples with free neutrophil elastase (+NE) that was often accompanied by decreased density of the $\sim 92-\mathrm{kDa}$ isoform. Representative samples are shown with purified neutrophil elastase (lane 7) and recombinant human MMP-9 (lane 8). d) Uninfected BALF; e) infected BALF. 

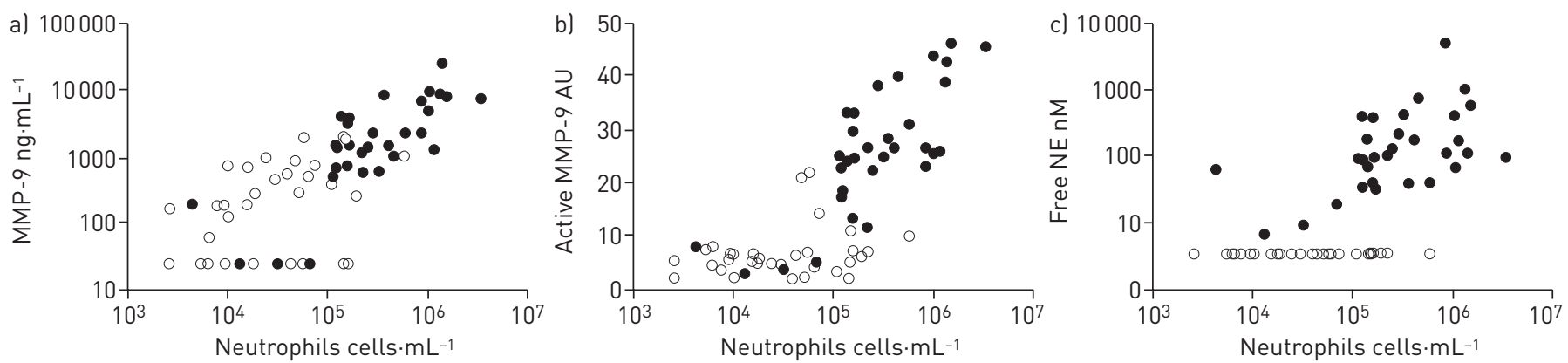

FIGURE 2 Neutrophil infiltration is associated with matrix metalloproteinase (MMP)-9 and free neutrophil elastase (NE) in bronchoalveolar lavage fluid. Shaded symbols represent samples with detectable free NE. a) The relationship between total MMP-9 and neutrophil number. Spearman $\mathrm{r}=0.757$, $\mathrm{p}<0.0001$. b) The relationship between active MMP-9 and neutrophil number. Spearman $r=0.752, p<0.0001$. c) The relationship between free NE and neutrophil number. Spearman $\mathrm{r}=0.701, \mathrm{p}<0.0001$. AU: arbitrary units.

Changes in MMP expression and activation were also assessed on a single-pathogen basis for the two most common pathogens in the group, Aspergillus species and $P$. aeruginosa (online supplementary table S2). Airways with Aspergillus species present demonstrated significant increases in total MMP-9 (median 1388 versus $4696 \mathrm{ng} \cdot \mathrm{mL}^{-1}, \mathrm{p}=0.0109$ ), MMP-9/TIMP-1 ratio (median 34.02 versus $85.50, \mathrm{p}=0.0076$ ), as well as active MMP-9 (median 7.15 versus $25.00 \mathrm{AU}, \mathrm{p}=0.0092$ ) (table S2). However, there were no significant changes observed when $P$. aeruginosa was detected, despite significantly increased free neutrophil elastase activity when either organism was present (Aspergillus median 3.39 versus $108.10 \mathrm{nM}, \mathrm{p}=0.0107$; $P$. aeruginosa median 3.39 versus $121.20 \mathrm{nM}, \mathrm{p}=0.0335$ ) (table S2).

Since neutrophils are a potent source of MMP-9, the association between concentration of neutrophils in BALF and MMP-9 was assessed (fig. 2). Neutrophil number per mL BALF significantly correlated with total MMP-9 $(\mathrm{r}=0.76, \mathrm{p}<0.0001)$ (fig. 2a), which was further confirmed by a nonlinear, monotonic relationship ( $\mathrm{r}=0.54, \mathrm{p}<0.0001)$ to MMP-9/TIMP ratio (not shown). A similar relationship to neutrophil number was observed for active MMP-9 ( $\mathrm{r}=0.75, \mathrm{p}<0.0001)$ (fig. $2 \mathrm{~b}$ ). A significant increase in active MMP-9 occurred when neutrophil number exceeded $1 \times 10^{5}$ cells $\mathrm{mL}^{-1}(6.50 \pm 4.88$ versus $23.88 \pm 12.58 \mathrm{AU}$, $\mathrm{p}<0.0001$ ), with detectable free neutrophil elastase also more prevalent during neutrophilia (fig. $2 \mathrm{c}$ ). In contrast, no significant associations were seen between concentration of macrophages and either MMP-9 or neutrophil elastase (online supplementary fig. S1). When the relationships of active MMP (fig. 3a) and active MMP-9/TIMP-1 ratio (fig. 3b) to neutrophil elastase concentration were assessed directly, both were found to be statistically significant $(r=0.79, \mathrm{p}<0.0001$ and $\mathrm{r}=0.69, \mathrm{p}<0.0001$, respectively).

In order to address the hypothesis that MMP-2 and MMP-9 profiles in the airways are associated with disease severity, we first examined samples stratified into four disease classes, based upon the interaction of infection and presence of free neutrophil elastase (fig. 4), as follows. No current infection and no free neutrophil elastase (class 1); no current infection but free neutrophil elastase detected (class 2); infection but no free neutrophil elastase (class 3); and infection with free neutrophil elastase detected (class 4). Mean MMP-2/TIMP-2 ratio (fig. 4a) varied significantly between classes, with a lower ratio observed in BALF with both infection and free neutrophil elastase (class 4) in comparison to BALF with neither
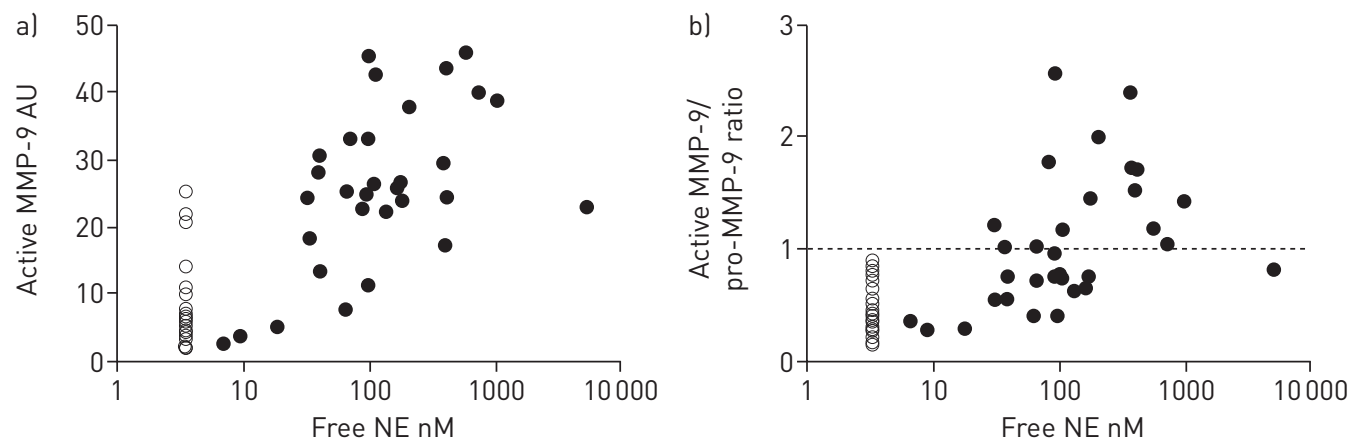

FIGURE 3 Free neutrophil elastase (NE) correlates with activation of matrix metalloproteinase (MMP)-9 in bronchoalveolar lavage fluid. Shaded symbols represent samples with detectable free NE. a) The relationship between active MMP-9 and free NE. Spearman $\mathrm{r}=0.787, \mathrm{p}<0.0001$. b) The relationship between active MMP-9/pro-enzyme MMP-9 (pro-MMP-9) ratio and free NE. Spearman $r=0.678, p<0.0001$. Dotted line represents equal ratio of active and inactive MMP-9. Area above dotted line indicates active MMP-9. AU: arbitrary units. 

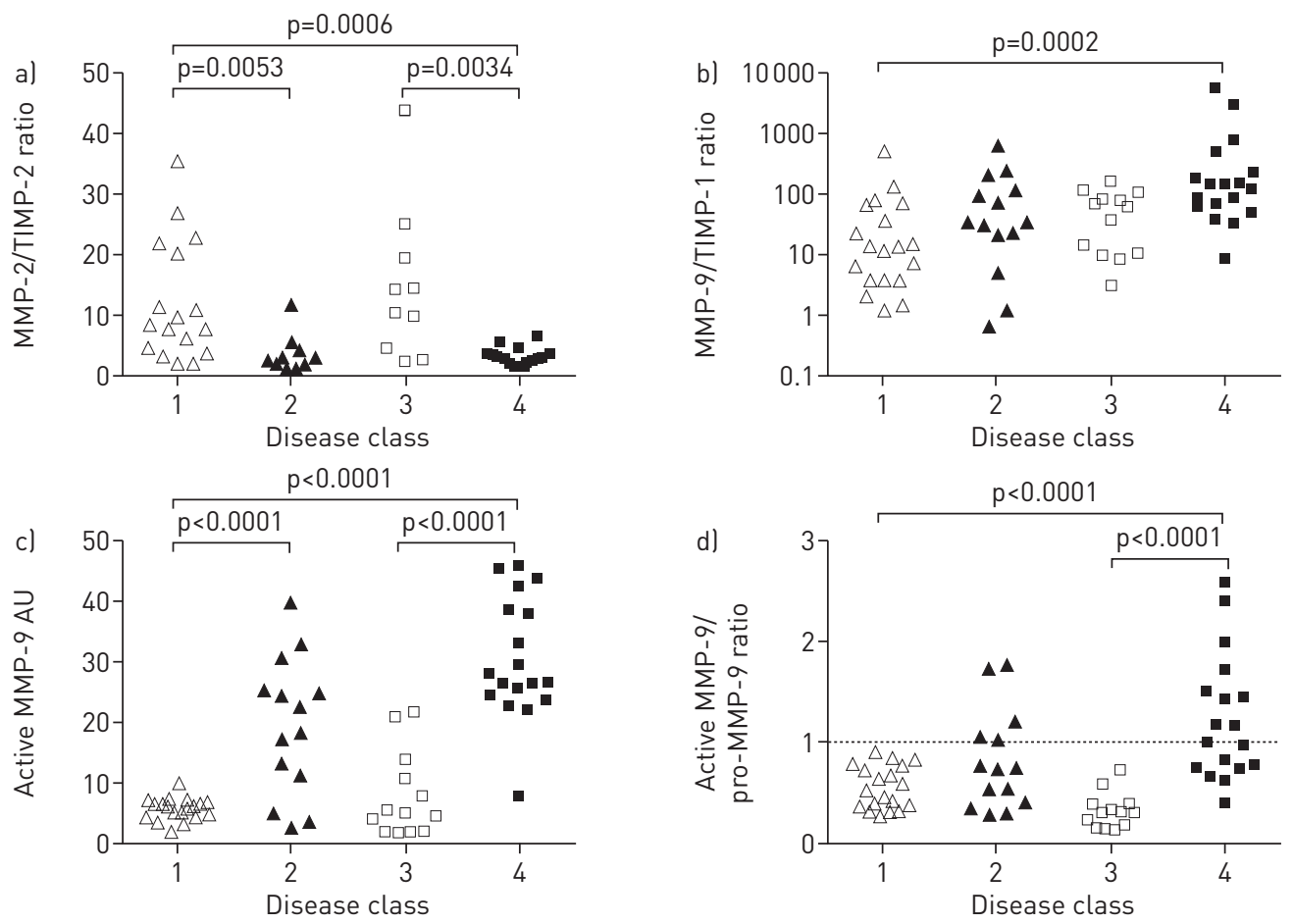

FIGURE 4 Matrix metalloproteinase (MMP) regulation changes with disease severity in bronchoalveolar lavage fluid with (squares) and without (triangles) current infection. Shaded symbols represent samples with detectable free neutrophil elastase (NE) activity. a) MMP-2/tissue inhibitor of matrix metalloproteinase (TIMP)-2 ratio by disease class. b) MMP-9/TIMP-1 ratio by disease class. c) Active MMP-9 (AU: arbitrary units) by disease class. d) Active MMP-9/ pro-enzyme MMP-9 (pro-MMP-9) ratio by disease class. Dotted line represents equal ratio of active and inactive MMP-9. Area above dotted line indicates active MMP-9. Class 1: no current infection, no NE activity ( $\mathrm{n}=19)$; class 2: no current infection, free NE $(n=14)$; class 3: infected, no NE ( $n=13)$; class 4: infected, free $N E(n=18)$.

infection nor free neutrophil elastase (class 1) (2.96 \pm 1.45 versus $11.76 \pm 9.84$, respectively; $\mathrm{p}<0.01)$. Although mean MMP-9/TIMP-1 ratio (fig. 4b) was only increased in class 4 (49.72 \pm 110.10 versus 605.20 $\pm 1386.00, \mathrm{p}<0.001$ ), active MMP-9 was consistently elevated $(\mathrm{p}<0.0001)$ (fig. $4 \mathrm{c}$ ) in the presence of free neutrophil elastase (classes 1 and 4) compared to BALF with no free neutrophil elastase. Mean levels of active MMP-9 in class 4 were five-fold higher than in class 1 (30.75 \pm 10.0 versus $65.80 \pm 1.81 \mathrm{AU}$, respectively; $\mathrm{p}<0.0001$ ), which also led to a significant increase in active MMP-9/pro-MMP-9 ratio (fig. $4 \mathrm{~d})$ between these disease classes $(0.56 \pm 0.21$ versus $1.24 \pm 0.62, \mathrm{p}<0.0001)$.

Finally, the relationships between MMP-9 and degree of structural lung disease were assessed by comparison to extent of bronchiectasis (fig. 5). A significant, positive relationship $(r=0.50, p<0.0001)$ was observed between MMP-9/TIMP-1 ratio and current extent of bronchiectasis (fig. 5a), as well as the change in extent $(\mathrm{r}=0.38, \mathrm{p}=0.0043)$ from the prior annual CT scan (fig. 5b). Weaker but significant correlations were also seen for active MMP-9 and both current bronchiectasis $(r=0.48, p=0.0002)$ and change in extent of bronchiectasis $(r=0.29, \mathrm{p}=0.0314)$.

\section{Discussion}

Our observations support the hypothesis that MMP-9 activity in the airways of very young children with cystic fibrosis is associated with disease severity. Furthermore, the observations from this unique cohort provide in vivo evidence that activation of the pro-MMP-9 zymogen is dependent upon the presence of free neutrophil elastase. Significantly, the strong correlation between dysregulated airway MMP-9 activity and extent of bronchiectasis suggests a possible nexus between neutrophil elastase activity in the airway and structural disease. Collectively, these findings provide a rationale for potential anti-protease therapies in the early stages of cystic fibrosis.

With the exception of MMP-7, MMPs are not typically observed in healthy tissue, as their primary function is to assist in mediating repair $[8,27]$. Therefore, detection of MMPs is indicative of airway disease, with elevated MMP-9/TIMP-1 ratios linked to worse outcomes in cystic fibrosis across a number of studies $[12,13,15,28]$. In this assessment of 64 BALF samples taken when children were free of exacerbation, but exhibiting a range of disease severity, the concentrations of MMP-2, MMP-9 and TIMP-1 reported are 

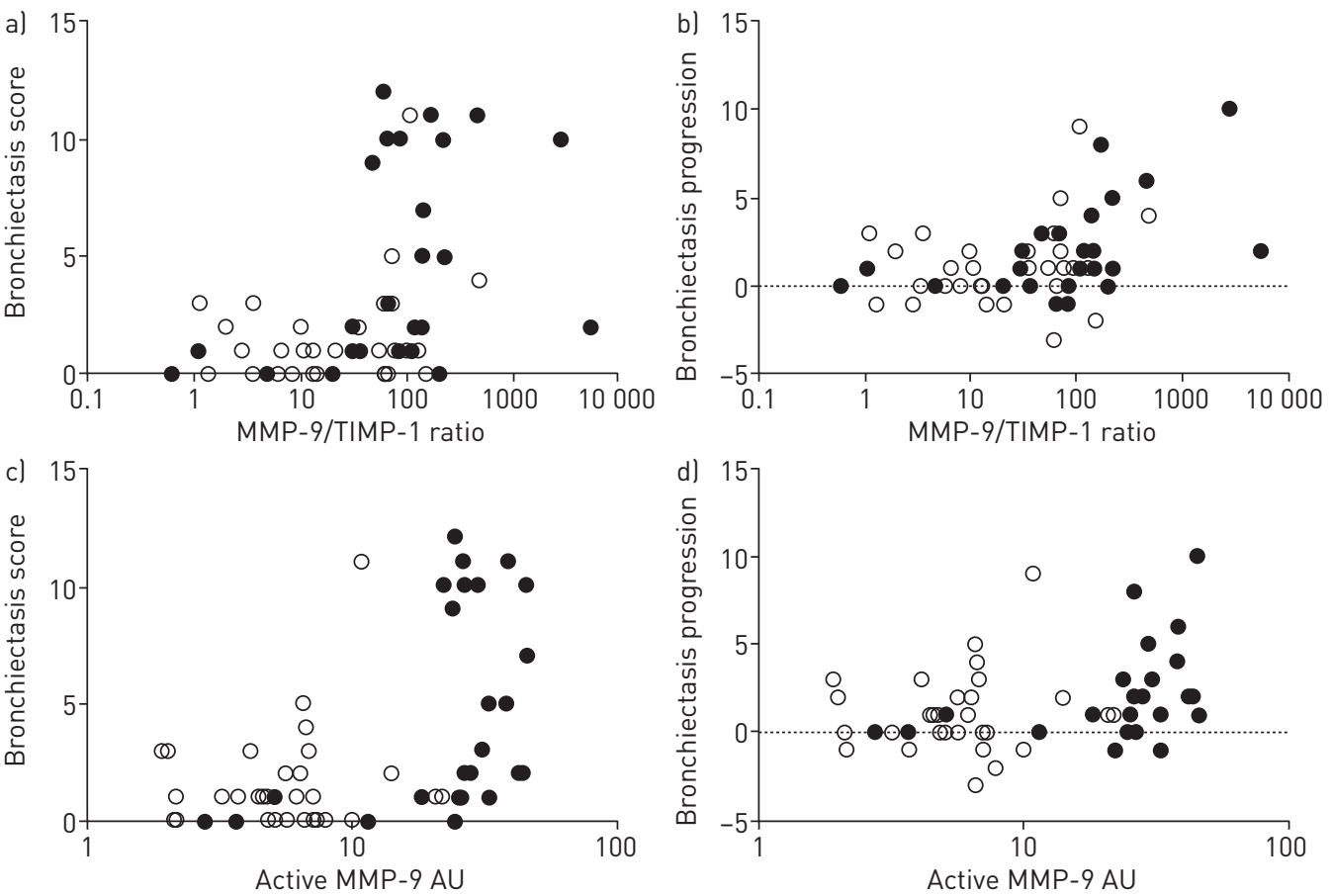

FIGURE 5 Uninhibited matrix metalloproteinase 9 (MMP-9) is associated with increased bronchiectasis. Shaded symbols represent samples with detectable free neutrophil elastase (NE) activity. a) The relationship between current bronchiectasis score and MMP-9/tissue inhibitor of matrix metalloproteinase 1 (MMP-9/TIMP-1) ratio. Spearman $r=0.496, p<0.0001 . b$ ) The relationship between change in bronchiectasis score over 12 months and MMP-9/TIMP-1 ratio. Spearman $\mathrm{r}=0.383$, $\mathrm{p}=0.0043$. Dotted line represents no change in bronchiectasis score. Area above dotted line indicates bronchiectasis has increased since previous scan. c) The relationship between current bronchiectasis score and active MMP-9 (AU: arbitrary units). Spearman $\mathrm{r}=0.479, \mathrm{p}=0.0002$. d) The relationship between change in bronchiectasis score over 12 months and active MMP-9. Spearman $\mathrm{r}=0.293$, $\mathrm{p}=0.0314$. Dotted line represents no change in bronchiectasis score. Area above dotted line indicates bronchiectasis has increased since previous scan.

similar to the minimal data available in children with cystic fibrosis aged $<6$ years [14, 19]. Ominously, 50 children exhibited a MMP-9/TIMP-1 ratio $>10$, and this was seen as early as 3 months of age. Increases in the MMP-9/TIMP-1 ratio were predominantly due to increases in total MMP-9, with an insufficient increase in TIMP-1 release observed. A recent study reported that plasma TIMP-1 concentration was adversely associated with survival in adults [15]. These counterintuitive findings suggest that the innate regulatory mechanisms of MMP-9 fail to respond correctly in the lung, and, although not assessed in this study, neutrophil elastase mediated degradation of TIMP-1 may contribute to elevated MMP/TIMP-1 in children with elevated neutrophil elastase in BALF $[13,18]$. Finally, the strong correlation with neutrophil number indicates that MMP-9 is predominantly derived from neutrophils. While reduced release of MMP-9 by cystic fibrosis neutrophils due to poor degranulation has been reported [29], no difference was observed in the total MMP-9 carried within each neutrophil. Excessive neutrophilia is a hallmark of cystic fibrosis due to a hyperinflammatory epithelial response [30,31], and therefore any small differences in MMP release is likely to be masked by the large cell numbers involved.

Increased activation of MMP-9 was only apparent in association with free detectable neutrophil elastase in BALF, providing a unique in vivo confirmation of the neutrophil elastase-driven model of pathophysiology proposed by JACKSON et al. [18]. Free neutrophil elastase has been recently identified as a predictor of bronchiectasis in infants with cystic fibrosis [5] and a recent report of significantly reduced lung disease when neutrophil elastase is genetically deleted in a mouse model of cystic fibrosis further strengthens this hypothesis [32]. This study is the first to report that increased MMP-9/TIMP-1 and active MMP-9 ratios were significantly correlated with progression of bronchiectasis over the previous year. Both neutrophil elastase and MMP-9 have considerable capability to modify tissue structure, thus it is feasible that development of a chronic proteolytic load is likely to contribute directly to the persistent, progressive airway remodelling observed [33]. However, key questions remain: how much MMP activation is required during lung repair and whether chronic activation of MMP is disruptive to these processes.

We observed that MMP-2 expression decreased in the presence of neutrophil elastase and the data presented here are supported by previous reports of minimal or absent concentrations of MMP-2 in lower 
airway secretions in cystic fibrosis $[13,19]$. This may be due to neutrophil elastase-mediated degradation to which MMP-9 appears to be resistant [16-18]. Alternatively, reports of decreased serum MMP-2 concentration during cystic fibrosis exacerbations suggest that this phenomenon may in fact be a regulatory response [28]. Our cross-sectional observations indicate that MMP pathways are hyperactivated when neutrophil elastase is not adequately inhibited. Future longitudinal studies to investigate changes in MMP and TIMP expression over time should further elaborate their contribution to structural damage and the possibility of their use as biomarkers. Although the minimal expression of MMP-1 and MMP-7 in these samples limits any understanding of their role in early cystic fibrosis, these proteases and MMP-12 are believed to play important roles in the re-epithelialisation of tissues following injury and can be disrupted in chronic lung diseases, including cystic fibrosis [7, 28, 34, 35]. A more recent study identified that MMP-12 contributes to structural lung abnormalities in a mouse model of cystic fibrosis, with deletion of MMP-12 at the gene level reducing emphysema [36]. This study also demonstrated that macrophages in BALF from patients with cystic fibrosis had elevated MMP-12 expression compared to healthy controls. Of interest was the observation that patients with minor functional polymorphisms in the MMP-12 gene promoter region were protected from lower lung function compared to patients with wild-type alleles [36]. Therefore, future studies assessing the relationship of these additional MMPs to structural changes would further benefit the understanding of their role in early cystic fibrosis disease. These novel data also indicate that more sensitive techniques are required to assess nongelatinase MMPs in BALF from this age cohort. However, the data on MMP-2 and MMP-9 from this study do suggest that even in asymptomatic individuals, the observation of free neutrophil elastase activity should be considered an important precursor to cystic fibrosis structural disease and one that requires a therapeutic response.

Antiprotease therapies have not been a focus for interventions in children with cystic fibrosis. Instead, inflammation has been targeted by limiting inflammatory triggers through antibacterial prophylaxis, reducing mucous retention in the airways, and direct anti-inflammatory therapies, such as ibuprofen. These interventions might reduce the protease burden indirectly by reducing neutrophilic inflammation. Yet there is accumulating evidence that direct antiprotease therapies should now be considered in an attempt to prevent progression of structural lung disease [5]. Overlapping substrate specificities pose problems for inhibiting specific MMPs [9, 37], so targeting neutrophil elastase offers the shortest translation route for minimising protease burden by inhibiting neutrophil elastase and reducing MMP-9 activation. Preferentially targeting the inhibition of free neutrophil elastase activity is also preferable, since many other biological processes can be dysregulated by neutrophil elastase, including the complement pathway for bacterial killing and mucous hypersecretion [38, 39]. Inhibition could be achieved by supplementing endogenous levels of $\alpha_{1}$-antitrypsin. Although safe in adults [40], $\alpha_{1}$-antitrypsin therapies have not yet been trialled in children with cystic fibrosis aged $<8$ years, despite a lack of obvious complications, and the early establishment of lung disease in cystic fibrosis warranting therapeutic assessment.

In summary, this study has demonstrated that even in early-life cystic fibrosis, expression patterns of MMPs change significantly in the presence of infection and free neutrophil elastase. These proteomic changes occur despite relatively mild cystic fibrosis disease and are associated with structural disease. Therefore, as evidence linking neutrophil infiltration and establishment of cystic fibrosis lung disease continues to grow, the clinical response to early inflammation may need to be reconsidered. Since MMPs are likely to play a critical role in the abnormal remodelling of cystic fibrosis airways, targeting the inhibition of free neutrophil elastase in cystic fibrosis could disrupt the pathway that links neutrophil inflammation and the early development of bronchiectasis.

\section{Acknowledgements}

The members of the Australian Respiratory Early Surveillance Team for Cystic Fibrosis (AREST CF) are: G. Banton, L. Bennett, L. Berry, C. Branch-Smith, A. Chong, N. De Klerk, R. Foong, L. Garratt, G. Hall, A. Harper, L. Hughes, A. Kicic, I. Laing, L. McCahon, A-M. Naylor, P. Noakes, M. Padros, K. Ramsey, T. Rosenow, N. Shaw, P. Sly, E. Sutanto, L. Turkovic, A. Williams (Telethon Kids Institute, University of Western Australia, Perth, Australia); A-M. Adams, K. Azzopardi, J. Carlin, R. Carzino, N. Clarke, D. Galadis, L. King, K. Logie, J. Sheehan, B. Skoric, J. Smith, P. Sutton, S. Vidmar (Murdoch Children's Research Institute, Melbourne, Australia); E. Balding, B. Clements, L. Mott, C. Murray, S. Oo, L. Owens, R. Puvvadi, S. Stick (Dept of Respiratory Medicine, Princess Margaret Hospital for Children, Perth, Australia); and J. Ajzner, K. Frayman, P. Griffin, J. Harrison, J. Massie, S. Ranganathan, P. Robinson, C. Robertson (Dept of Respiratory Medicine, Royal Children's Hospital, Melbourne, Australia).

The authors would like to thank Luke Berry (Telethon Kids Institute) for his technical assistance in processing bronchoalveolar lavage fluid and the interleukin- 8 and neutrophil elastase assays, as well as Colin Robertson (Royal Children's Hospital, Melbourne), Graham Hall (Telethon Kids Institute) and Lauren Mott (Princess Margaret Hospital for Children, Perth) for their comments during manuscript preparation. AREST CF would like to thank the contributions of respiratory fellows and nurses at Princess Margaret Hospital for Children, Telethon Kids Institute, Royal Children's Hospital and Murdoch Children's Research Institute. We would also like to thank the participants and their families who contribute to the AREST CF programme. 


\section{References}

1 Mahadeva R, Webb K, Westerbeek RC, et al. Clinical outcome in relation to care in centres specialising in cystic fibrosis: cross sectional study. BMJ 1998; 316: 1771-1775.

2 Sly PD, Brennan S, Gangell C, et al. Lung disease at diagnosis in infants with cystic fibrosis detected by newborn screening. Am J Respir Crit Care Med 2009; 180: 146-152.

3 Stick SM, Brennan S, Murray C, et al. Bronchiectasis in infants and preschool children diagnosed with cystic fibrosis after newborn screening. J Pediatr 2009; 155: 623-628.

4 Armstrong DS, Hook SM, Jamsen KM, et al. Lower airway inflammation in infants with cystic fibrosis detected by newborn screening. Pediatr Pulmonol 2005; 40: 500-510.

5 Sly PD, Gangell CL, Chen L, et al. Risk factors for bronchiectasis in children with cystic fibrosis. $N$ Engl J Med 2013; 368: 1963-1970.

6 Voynow JA, Fischer BM, Zheng S. Proteases and cystic fibrosis. Int J Biochem Cell Biol 2008; 40: 1238-1245.

7 Gaggar A, Hector A, Bratcher PE, et al. The role of matrix metalloproteinases in cystic fibrosis lung disease. Eur Respir J 2011; 38: 721-727.

8 Löffek S, Schilling O, Franzke C-W. Biological role of matrix metalloproteinases: a critical balance. Eur Respir J 2011; 38: 191-208.

9 Maskos K, Bode W. Structural basis of matrix metalloproteinases and tissue inhibitors of metalloproteinases. Mol Biotechnol 2003; 25: 241-266.

10 Delacourt C, Le Bourgeois M, D’Ortho MP, et al. Imbalance between 95kDa type IV collagenase and tissue inhibitor of metalloproteinases in sputum of patients with cystic fibrosis. Am J Respir Crit Care Med 1995; 152: 765-774.

11 Ratjen F, Hartog C-M, Paul K, et al. Matrix metalloproteases in BAL fluid of patients with cystic fibrosis and their modulation by treatment with dornase alpha. Thorax 2002; 57: 930-934.

12 Sagel SD, Kapsner RK, Osberg I. Induced sputum matrix metalloproteinase-9 correlates with lung function and airway inflammation in children with cystic fibrosis. Pediatr Pulmonol 2005; 39: 224-232.

13 Gaggar A, Li Y, Weathington N, et al. Matrix metalloprotease-9 dysregulation in lower airway secretions of cystic fibrosis patients. Am J Physiol Lung Cell Mol Physiol 2007; 293: L96-L104.

14 Hilliard TN, Regamey N, Shute JK, et al. Airway remodelling in children with cystic fibrosis. Thorax 2007; 62: 1074-1080.

15 Devereux G, Steele S, Jagelman T, et al. An observational study of matrix metalloproteinase (MMP)-9 in cystic fibrosis. I Cyst Fibros 2014; 13: 557-563

16 Okada Y, Nakanishi I. Activation of matrix metalloproteinase 3 (stromelysin) and matrix metalloproteinase 2 ('gelatinase') by human neutrophil elastase and cathepsin G. FEBS Lett 1989; 249: 353-356.

17 Shamamian P, Schwartz JD, Pocock BJ, et al. Activation of progelatinase A (MMP-2) by neutrophil elastase, cathepsin G, and proteinase-3: a role for inflammatory cells in tumor invasion and angiogenesis. J Cell Physiol 2001; 189: 197-206.

18 Jackson PL, Xu X, Wilson L, et al. Human neutrophil elastase-mediated cleavage sites of MMP-9 and TIMP-1: implications to cystic fibrosis proteolytic dysfunction. Mol Med 2010; 16: 159-166.

19 Peterson-Carmichael SL, Harris WT, Goel R, et al. Association of lower airway inflammation with physiologic findings in young children with cystic fibrosis. Pediatr Pulmonol 2009; 44: 503-511.

20 De Blic J, Midulla F, Barbato A, et al. Bronchoalveolar lavage in children. ERS Task Force on bronchoalveolar lavage in children. European Respiratory Society. Eur Respir J 2000; 15: 217-231.

21 Berry LJ, Sheil B, Garratt L, et al. Stability of interleukin 8 and neutrophil elastase in bronchoalveolar lavage fluid following long-term storage. J Cyst Fibros 2010; 9: 346-350.

22 Brennan S, Sly PD, Gangell CL, et al. Alveolar macrophages and CC chemokines are increased in children with cystic fibrosis. Eur Respir J 2009; 34: 655-661.

23 Banerjee B, Ling K-M, Sutanto EN, et al. The airway epithelium is a direct source of matrix degrading enzymes in bronchiolitis obliterans syndrome. J Heart Lung Transplant 2011; 30: 1175-1185.

24 Rossano R, Larocca M, Riviello L, et al. Heterogeneity of serum gelatinases MMP-2 and MMP-9 isoforms and charge variants. J Cell Mol Med 2014; 18: 242-252.

25 Bellini T, Trentini A, Manfrinato MC, et al. Matrix metalloproteinase-9 activity detected in body fluids is the result of two different enzyme forms. J Biochem 2012; 151: 493-499.

26 Snoek-van Beurden PAM, Von den Hoff JW. Zymographic techniques for the analysis of matrix metalloproteinases and their inhibitors. Biotechniques 2005; 38: 73-83.

27 Murphy G, Nagase H. Progress in matrix metalloproteinase research. Mol Aspects Med 2008; 29: 290-308.

28 Roderfeld M, Rath T, Schulz R, et al. Serum matrix metalloproteinases in adult CF patients: relation to pulmonary exacerbation. J Cyst Fibros 2009; 8: 338-347.

29 Pohl K, Hayes E, Keenan J, et al. A neutrophil intrinsic impairment affecting Rab27a and degranulation in cystic fibrosis is corrected by CFTR potentiator therapy. Blood 2014; 124: 999-1009.

30 Sutanto EN, Kicic A, Foo CJ, et al. Innate inflammatory responses of pediatric cystic fibrosis airway epithelial cells: effects of nonviral and viral stimulation. Am J Respir Cell Mol Biol 2011; 44: 761-767.

31 Beaudoin T, LaFayette S, Roussel L, et al. The level of p38 $\alpha$ mitogen-activated protein kinase activation in airway epithelial cells determines the onset of innate immune responses to planktonic and biofilm Pseudomonas aeruginosa. J Infect Dis 2013; 207: 1544-1555.

32 Gehrig S, Duerr J, Weitnauer M, et al. Lack of neutrophil elastase reduces inflammation, mucus hypersecretion, and emphysema, but not mucus obstruction, in mice with cystic fibrosis-like lung disease. Am J Respir Crit Care Med 2014; 189: 1082-1092.

33 Mott LS, Park J, Murray CP, et al. Progression of early structural lung disease in young children with cystic fibrosis assessed using CT. Thorax 2012; 67: 509-516.

34 Hajj R, Lesimple P, Nawrocki-Raby B, et al. Human airway surface epithelial regeneration is delayed and abnormal in cystic fibrosis. J Pathol 2007; 211: 340-350.

35 Dunsmore SE, Saarialho-Kere UK, Roby JD, et al. Matrilysin expression and function in airway epithelium. J Clin Invest 1998; 102: 1321-1331.

36 Trojanek JB, Cobos-Correa A, Diemer S, et al. Airway mucus obstruction triggers macrophage activation and matrix metalloproteinase 12-dependent emphysema. Am J Respir Cell Mol Biol 2014; 51: 709-720. 
37 Vandenbroucke RE, Dejonckheere E, Libert C. A therapeutic role for matrix metalloproteinase inhibitors in lung diseases? Eur Respir J 2011; 38: 1200-1214.

38 Tosi MF, Zakem H, Berger M. Neutrophil elastase cleaves C3bi on opsonized Pseudomonas as well as CR1 on neutrophils to create a functionally important opsonin receptor mismatch. J Clin Invest 1990; 86: 300-308.

39 Voynow JA, Young LR, Wang Y, et al. Neutrophil elastase increases MUC5AC mRNA and protein expression in respiratory epithelial cells. Am J Physiol 1999; 276: L835-L843.

40 Griese M, Latzin P, Kappler M, et al. $\alpha_{1}$-Antitrypsin inhalation reduces airway inflammation in cystic fibrosis patients. Eur Respir J 2007; 29: 240-250. 